\title{
THE INVOLUTION CURVE DETERMINED FROM A SPECIAL PENCIL OF $n$-ICS*
}

\author{
BY \\ JOSEPHINE H. CHANLER
}

Introduction. In The geometry of the Weddle manifold $W_{p}[1] \dagger$ there arises the necessity for investigating involution curves, some of which cover $W_{p}$, while others cover the sections of $W_{p}$ by its $F$-loci. Since the points of an involution curve $V_{k}$ are in $(1,1)$ correspondence with the $k$-ads from the members of a pencil of $n$-ics, $V_{k}$ may be constructed as follows: Assign the parameter $t$ to the points of a norm curve $N^{k}$ in $S_{k}$. The hyperplanes of the curve given by a general member of the pencil intersect in $C_{n, k}$ points, the locus of which is the curve $V_{k}$ of order $C_{n-1, k-1}$. The involution curves of $W_{p}$ are particularized by the fact that the pencil of $n$-ics contains one member with a number of double roots. In the study of these it is important to determine the genus; it is likewise necessary to discover what multiple points are introduced by the special $n$-ic if we are to ascertain the effect of the Cremona transformations associated with $W_{p}$. The article just cited makes no attempt to solve these problems except for special values of $n, k$. In the present paper $(\$ \$ 1-4)$ we solve them for general $n, k$ where one $n$-ic of the pencil possesses $j$ distinct double roots $(1 \leqq j \leqq n / 2)$.

We determine the genus of $V_{k}$ by applying to the correspondence set up between the points of $N^{k}$ and the points of $V_{k}$ Zeuthen's formula:

$$
\eta-\eta^{\prime}=2 \alpha\left(p^{\prime}-1\right)-2 \alpha^{\prime}(p-1) \text {. }
$$

Here $\alpha, \alpha^{\prime}$ are the indices of the correspondence, $p, p^{\prime}$ the genera of the curves, and $\eta, \eta^{\prime}$ the numbers of branch points of the curves. $\eta, \eta^{\prime}$ may be calculated in either of two ways. By the first method, the point $t_{1}$ of $N^{k}$ is counted $v_{1}-1$ times as a branch point if $v_{1}$ of the points $y$ of $V_{k}$ corresponding to $t_{1}$ coalesce at $\gamma$ on the same branch of $V_{k}$. Hence we must first investigate the multiple points of $V_{k}$. This method I use in $\$ 1$, and used originally in $\$ 2$. The second much more elegant method, which may be applied at once without a study of the multiple points, was pointed out to me by O. Zariski. It depends on the following criterion for the multiplicity of a coincidence in an $\left(\alpha, \alpha^{\prime}\right)$ correspondence between two algebraic curves $V$ and $V^{\prime}$ : Let $P_{0}$ be a point on the

* Presented to the Society, December 31, 1936; received by the editors March 27, 1936, and, in revised form, August 27, 1936.

$\dagger$ Numbers in brackets refer to the bibliography at the end of the paper. 
Riemann surface of $V$; as a variable point $P$ of $V$, very near $P_{0}$, turns around $P_{0}$, the corresponding $\alpha^{\prime}$ points on $V^{\prime}$ may be permuted among themselves. If this permutation consists of cycles of periods $v_{1}, v_{2}, \cdots, v_{m},\left(\sum_{i=1}^{m} v_{i}=\alpha^{\prime}\right)$, then to the point $P_{0}$ there corresponds on $V^{\prime}, m$ coincidences of multiplicities $v_{1}, v_{2}, \cdots, v_{m}$ respectively. $P_{0}$ then contributes to $\eta$ the value $\sum_{i=1}^{m}\left(v_{i}-1\right)$. By means of this method we readily study the special cases of $\$ 6$, where some or all of the $j$ double roots of the special $n$-ics coincide. It is interesting to note that $\eta, \eta^{\prime}$ are not always identical for the two definitions, though their difference is the same for each.

In $\S 3$ we determine the multiple points of $V_{k}$ for $j>1$ by comparing the value of its genus as obtained in $\$ 2$ to the value determined by the first method. In $\$ 4$ we check this result by a projective study of $V_{k}$ as part of the intersection $\Gamma$ of $k-1$ hypersurfaces. These hypersurfaces are represented by the vanishing of $k-1$ determinants of highest order selected in a special way from a matrix whose elements are binary $n$-ics. The order of the manifold represented by the vanishing of all the determinants of highest order in such a matrix has been studied by Brill [2] as the order of a restricted system of equations. One reason for the introduction of this second proof is the light that it throws upon such manifolds; in particular the machinery set up for our work shows that Brill's formulas may be more easily obtained from the study of a dual matrix whose elements are linear forms in several variables. We thus prove that Brill's problem reduces to a special case of those investigated by Salmon, Roberts, Cayley, and Pieri. A more important reason for the projective method is that it introduces interesting manifolds associated with $V_{k}$; also it seems adapted to the study of manifolds which are obtained from $V_{k}$ by generalization.

In $\$ 5$ we prove an interesting identity involving binomial coefficients which I have not found in the literature.

1. The involution curve of the general pencil. The pencil of $n$-ics $\left[\left(a_{1} t\right)^{n},\left(a_{2} t\right)^{n}\right]$ contains $2(n-1)$ members with a double root each. We have for $N^{k}, V_{k}$ :

$$
\alpha=k, \quad \alpha^{\prime}=C_{n-1, k-1}, \quad p=0, \quad p^{\prime}=p_{k} .
$$

Since the involution curve has no multiple points, we easily calculate $\eta, \eta^{\prime}$ by the first method:

$$
\eta=2(n-1)(k-1) C_{n-2, k-1} ; \quad \eta^{\prime}=2(n-1) C_{n-2, k-2} .
$$

Substituting these values in Zeuthen's formula, we have

$$
p_{k}=(n-k) C_{n-1, k-1}-C_{n, k}+1 \text {. }
$$


This is unaltered when $k$ and $n-k$ are interchanged, checking the fact that $k$-ads and complementary $(n-k)$-ads in the pencil of $n$-ics determine two curves in $(1,1)$ correspondence.

2. The genus of the involution curve $V_{k}$ for which one $n$-ic possesses several double roots. Let the pencil contain one $n$-ic with $j$ distinct double roots, while there are $2(n-1)-j n$-ics with a double root each. Since the $n$-ics of the latter type behave as before with respect to $\eta$, $\eta^{\prime}$, we have for these

$$
\eta_{0}=(2 n-2-j)(k-1) C_{n-2, k-1}, \quad \eta_{0}^{\prime}=(2 n-2-i) C_{n-2, k-2}
$$

so that

$$
\eta_{0}-\eta_{0}^{\prime}=(2 n-2-j)(n-k-1) C_{n-2, k-2} .
$$

To determine the special $n$-ic's contributions $\eta_{s}, \eta_{s}^{\prime}$ to $\eta$, $\eta^{\prime}$, we use Zariski's argument.

Let the special $n$-ic of the pencil correspond to the value $x_{0}$ of the parameter $x$. As $x$ very near $x_{0}$ turns around $x_{0}$, the roots of the $n$-ic corresponding to $x$ will be permuted, the permutation consisting of $j$ cycles of period two and $n-2 j$ cycles of period one. For convenience we write this permutation

$$
S=\left(t_{11} t_{12}\right) \cdots\left(t_{i 1} t_{i 2}\right) \cdots\left(t_{j 1} t_{j 2}\right)\left(\tau_{1}\right)\left(\tau_{2}\right) \cdots\left(\tau_{n-2}\right) .
$$

A corresponding permutation $\Sigma$ takes place among the $k$-ads of $t$ 's, $\tau$ 's chosen from the roots of the $n$-ic. As $x$ approaches $x_{0}$, a letter of the $i$ th cycle approaches the point $P_{i}$ of $N^{k}$ corresponding to the double root of the special $n$-ic, and $\tau_{h}$ approaches the point $Q_{h}$ of $N^{k}$. The $k$-ads of $t$ 's and $\tau$ 's approach points $\pi(t, \tau)$ of $V_{k}$. To find the number of coincidences on $V_{k}$ corresponding to $P_{i}$ or $Q_{h}$, we let $t_{i}$ or $\tau_{h}$ turn around $P_{i}$ or $Q_{h}$, and write down the corresponding permutation on the $k$-ads involving $t_{i}$ or $\tau_{h}$. From this it is seen that the $P_{i}$ are not branch points, since the corresponding permutations are induced by $\Sigma^{2}$, and $\Sigma$ is of period two. The permutations corresponding to $Q_{h}$ are induced by $\Sigma$, under which the $k$-ads actually permuted are those and only those which contain one only of some pair $\left(t_{i 1} t_{i 2}\right)$. Such a $k$-ad is interchanged with another as $\tau_{h}$ turns about $Q_{h}$. The total number of $k$-ads containing one and only one point from each of $r$ pairs $\left(t_{i 1}, t_{i 2}\right), 1 \leqq r \leqq j$, and $k-r-2 l$ points $\tau_{h}, 0 \leqq l \leqq j-r$, is $C_{j, r} C_{j-r, l} C_{n-2 j, k-r-2 l} 2^{r}$. Since each such $k$-ad contains $k-r-2 l$ branch points, and since the $k$-ads are permuted in pairs by $\Sigma$, we have

$$
\eta_{s}=\sum_{r=1}^{j} \sum_{l=0}^{j-r} C_{j, r} C_{j-r, l} C_{n-2 j, k-r-2 l}(k-r-2 l) 2^{r-1} .
$$

A point $\pi(t, \tau)$ of $V_{k}$, for which the $k$-ad turning about it contains one and 
only one point of a pair $\left(t_{i 1} t_{i 2}\right)$ cannot be a branch point, since the corresponding permutation among the $t^{\prime}$ s is induced by $S^{2}$. The $k$-ads containing both members of $l$ such pairs and $k-2 l$ points $\tau_{h}(1 \leqq l \leqq i)$ are $C_{j, l} C_{n-2 j, k-2 l}$ in number. Hence

$$
\eta_{s}^{\prime}=\sum_{l=1}^{j} C_{j, l} C_{n-2 j, k-2 l} l .
$$

The decrease in $p_{k}$ due to the presence of the special $n$-ic is therefore

and

$$
\begin{aligned}
\tilde{p}=\frac{1}{2 k}\{j(n-k-1) & C_{n-2, k-2}+\sum_{l=1}^{j} C_{j, l} C_{n-2 j, k-2 l} l \\
& \left.-\sum_{r=1}^{j} \sum_{l=0}^{j-r} C_{j, r} C_{j-r, l} C_{n-2 j, k-r-2 l}(k-r-2 l) 2^{r-1}\right\},
\end{aligned}
$$

$$
p_{k}=(n-k) C_{n-1, k-1}-C_{n, k}+1-\tilde{p} .
$$

3. The multiple points of $V_{k}$. The special $n$-ic introduces whatever multiple points exist. We determine what their nature must be to produce the value $\eta_{s}-\eta_{s}^{\prime}$ obtained from $\$ 2$, (3), (4). Let the special $n$-ic be $\left(t s_{1}\right)\left(t s_{1}\right)$ $\cdots\left(t s_{j}\right)\left(t s_{j}\right)\left(t t_{1}\right)\left(t t_{2}\right) \cdots\left(t t_{n-2}\right)$. Then the point $P_{l}\left(s_{1}, s_{1}, s_{2}, s_{2}, \cdots, s_{l}, s_{l}, t_{1}\right.$, $\left.t_{2}, \cdots, t_{k-2 l}\right)$ is a simple point on $V_{k}$ and contributes 0 to $\eta_{s}$ and $l$ to $\eta_{s}^{\prime}$. There are $\sum_{l=1}^{j} C_{j, l} C_{n-2 j, k-2 l}$ such points. At the point

$$
P_{r l}\left(s_{1}, s_{2}, \cdots, s_{r}, s_{r+1}, s_{r+1}, \cdots, s_{r+l}, s_{r+l}, t_{1}, t_{2}, \cdots, t_{k-r-2 l}\right)
$$

there are $2^{r-1}$ or fewer branches, since $s_{i}(i \leqq r)$ meets $V_{k}$ here in at most $2^{r-1}$ points. If there are $2^{r-1}$ branches, the contribution to $\eta_{s}$ due to the $t$ 's and the $s_{i}$ 's $(i>r)$ at the point is $(k-r-l) 2^{r-1}$; the $s_{i}$ 's $(i \leqq r)$ contribute nothing. On the other hand, the contribution to $\eta_{s}^{\prime}$ due to $P_{r l}$ is $l \cdot 2^{r-1}$. If the number of branches is smaller, the contributions to $\eta_{s}$ due to this point increase, while that to $\eta_{s}^{\prime}$ decreases. There are $\sum_{r=1}^{j} \sum_{l=0}^{j-r} C_{j, r} C_{j-r, l} C_{n-2 j, k-r-2 l}$ such points. Hence only if there are $2^{r-1}$ branches at each point $P_{r l}$, can we have

(1) $\eta_{s}-\eta_{s}^{\prime}=\sum_{r=1}^{j} \sum_{l=0}^{j-r} C_{j, r} C_{j-r, l} C_{n-2 j, k-r-2 l}(k-r-2 l) 2^{r-1}-\sum_{l=1}^{j} C_{j, l} C_{n-2 j, k-2 l} l$, the value obtained from $\$ 2,(3),(4)$.

TheOREM 1. The point $P_{r l}$ described above is a $2^{r-1}$-fold point on $V_{k}$; in the calculation of the genus it is equivalent to $(r-1) 2^{r-2}$ ordinary double points.

The first statement we have just proved. If the second holds, the decrease in the genus due to the special $n$-ic must be written: 


$$
\tilde{p}=\sum_{r=2}^{i} \sum_{l=0}^{j-r} C_{j, r} C_{j-r, l} C_{n-2 j, k-r-2 l}(r-1) 2^{r-2} .
$$

Equating the right-hand members of (2) and §2, (5) leads to the following, after simplification:

(3) $j(n-k-1) C_{n-2, k-2}=\sum_{r=0}^{j} \sum_{l=0}^{j-r} C_{j, r} C_{j-r, l} C_{n-2 j, k-r-2 l}(k r-r-2 l) 2^{r-1}$.

That (3) is satisfied under the conditions of our problem is proved in $\$ 5$.

4. The study of $V_{k}$ from a projective view point; its multiple points and the manifolds associated with it. Let the pencil of $n$-ics $\left[\left(a_{1} t\right)^{n},\left(a_{2} t\right)^{n}\right]$ be contained in a linear system $\Sigma_{k-1}=\left[\left(a_{1} t\right)^{n},\left(a_{2} t\right)^{n}, \cdots,\left(a_{k} t\right)^{n}\right]$. The $k$-ads of the pencil represent the points of a curve on the surface $M^{n-k+1}$ of order $n-k+1$ given by the equation

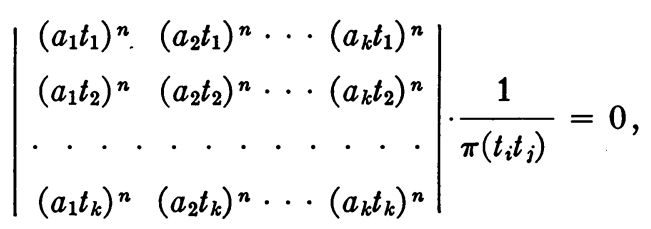

where $t_{1}, t_{2}, \cdots, t_{k}$ are interpreted as Darboux coordinates in the space $S_{k}$.

Take the matrix

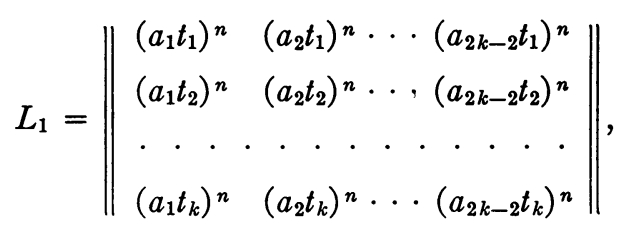

where the $n$-ics are linearly independent. Hence $k \leqq(n+3) / 2$; if not, we study instead the curve determined by the complementary $(n-k)$-ads. $L_{1}$ may be written briefly as $\left(a_{1} a_{2} \cdots a_{2 k-2}\right)$. From $L_{1}$ select any set of $k-1$ determinants such that the only columns common to all are $\left(a_{1} a_{2}\right)$, while each new determinant is obtained from that last found by dropping the third column and inserting as final column one not previously used. A typical set is $\left(a_{1} a_{2} \cdots a_{k}\right),\left(a_{1} a_{2} a_{4} \cdots a_{k+1}\right), \cdots,\left(a_{1} a_{2} a_{k+1} \cdots a_{2 k-2}\right)$. Such a set we define as a set $S$ of determinants.

The linearly independent $n$-ics $\left(a_{1} t\right)^{n}, \cdots,\left(a_{m} t\right)^{n}, m \leqq n+1$, determine $n+1$ linearly independent $n$-ics, $\left(c_{1} t\right)^{n},\left(c_{2} t\right)^{n}, \cdots,\left(c_{n+1} t\right)^{n}$, such that $\left(c_{i} t\right)^{n}$ is apolar to $\left(a_{j} t\right)^{n}, i \neq j$. In $S_{n}$ the hyperplane $\left(a_{j} t\right)^{n}$ is on the point $\left(c_{i} t\right)^{n}, i \neq j$. Thus the $k$-ad $\left(t_{1}, t_{2}, \cdots, t_{k}\right)$ making vanish the determinants of highest order of the matrix $\left(a_{1} a_{2} \cdots a_{m}\right), m \leqq k$, represents an $S_{k-1}$ (on the points $t_{1}, \cdots, t_{k}$ 
of the norm curve $\left.N^{k}\right)$ joined to the $S_{n-m}\left[\left(c_{m+1} t\right)^{n},\left(c_{m+2} t\right)^{n}, \cdots,\left(c_{n+1} t\right)^{n}\right]$ by an $S_{n-1}$. Hence the $S_{k-1}$ meets the $S_{n-m}$ in an $S_{k-m}$; that is, the $k$-ic $(q t)^{k}$ $=\left(t t_{1}\right)\left(t t_{2}\right) \cdots\left(t t_{k}\right)$ is apolar to $k-m+1$ independent members of the linear system $\left[\left(c_{m+1} t\right)^{n}, \cdots,\left(c_{n+1} t\right)^{n}\right]$. If $\sum_{i=m+1}^{n+1} \lambda_{i}\left(c_{i} q\right)^{k}\left(c_{i} t\right)^{n-k} \equiv 0$, we have $n-k+1$ equations in $n-m+1 \lambda$ 's. If there are $k-m+1$ independent solutions, the rank of the matrix of the system must be $n-k$. Thus the determinants of highest order in the matrix must vanish, which we indicate by writing $\left[c_{m+1} c_{m+2} \cdots c_{n+1}\right] \equiv 0$. The $k$-ad $\left(t_{1}, t_{2}, \cdots, t_{k}\right)$ making vanish the determinants of highest order of a matrix $\left(a_{1} a_{2} \cdots a_{m}\right), m>k$, represents an $S_{k-1}$ such that there are only $k-1$ conditions imposed for any hyperplane which is a linear combination of $\left(a_{1} t\right)^{n},\left(a_{2} t\right)^{n}, \cdots,\left(a_{m} t\right)^{n}$ to be on the $S_{k-1}$. The $S_{k-1}$ must therefore meet the $S_{n-m}\left[\left(c_{m+1} t\right)^{n},\left(c_{m+2} t\right)^{n}, \cdots,\left(c_{n+1} t\right)^{n}\right]$ in a point; that is, $(q t)^{k}=\left(t t_{1}\right)\left(t t_{2}\right) \cdots\left(t t_{k}\right)$ is apolar to a member of the linear system $\left[\left(c_{m+1} t\right)^{n},\left(c_{m+2} t\right)^{n}, \cdots,\left(c_{n+1} t\right)^{n}\right]$. As before, we have $n-k+1$ equations in $n-m+1 \lambda$ 's. For a solution to exist, the determinant must vanish for each set of $n-m+1$ equations. Thus again the matrix $\left[c_{m+1} c_{m+2} \cdots c_{n+1}\right] \equiv 0$. We note here that in either case the manifold represented by the matrix $\left(a_{1} a_{2} \cdots a_{m}\right) \equiv 0$, where the elements are binary $n$-ics, may likewise be represented by the matrix $\left[c_{m+1} c_{m+2} \cdots c_{n+1}\right] \equiv 0$, where the elements are linear in the coefficients of $(q t)^{k}$. Hence the formulas developed by Brill for the order of a manifold $\left(a_{1} a_{2} \cdots a_{m}\right) \equiv 0$ (cf. [2], pp. 391-395), may be more easily determined from the dual matrix $\left[c_{m+1} c_{m+2} \cdots c_{n+1}\right] \equiv 0$. For if we interpret the coefficients of $(q t)^{k}$ as variables, the latter becomes a matrix whose elements are linear forms. Such a matrix of $i$ rows and $j$ columns $(i \geqq j)$ is of order $C_{i, j-1}$ (cf. [3], p. 63).

We state without proof two preliminary theorems, the first of which is well known.

THEOREM 1. If in a matrix of $m$ rows and $n$ columns $(m<n)$ every determinant of the mth order containing a certain set of $i$ columns vanishes, while the $i$ columns are not linearly dependent, then every determinant of the mth order in the matrix vanishes.

THEOREM 2. The determinants of a set $S$ chosen as above from $L_{1}$, when divided by appropriate products and equated to zero, represent a set of hypersurfaces $M_{(s)}^{n-k+1}$ which intersect in a manifold $\Gamma_{s}$ of dimension one.

The truth of this theorem is implicitly assumed by Brill [2], though he does not use the geometric interpretation of this article. It may be checked by showing that the $t$ 's of a $k$-ad which represents a point on $\Gamma_{s}$ are the roots of a $k$-ic apolar to a point $n$-ic in each of the $S_{n-k}$ 's represented by the determinants of $S$; the $k-1$ conditions imposed on the coefficients of the $k$-ic 
can be proved linearly independent. One part of $\Gamma_{s}$ is the curve $V_{k}$. Another is the curve $V_{\Delta}$ whose points are represented by the $k$-ads making vanish all the determinants of highest order in $L_{1}$.

We now proceed to the fundamental theorems of this section.

THEOREM 3. No part of $V_{k}$ (except for a finite number of points) can appear multiply in any $\Gamma_{s}$.

If a point is of multiplicity two or more for the intersection curve of $k-1$ surfaces in $S_{k}$, while it is an ordinary point for each surface, the tangent hyperplanes of the surfaces at that point must have at least an $S_{2}$ in common (cf. [4], p. 191); that is, at most only $k-2$ tangent hyperplanes can be linearly independent. It may then be readily proved that at least one surface in the linear system determined by the $k-1 M_{(8)}^{n-k+1}$ must have a double point here. However, no surface which is a linear combination of the $M_{(8)}^{n-k+1}$ can have a multiple point at any point determined from a non-singular $n$-ic of the pencil.

THEOREM 4. There is no point on $V_{k}$ common to the curves residual to $V_{k}$ in all possible $\Gamma_{s}$ 's determined from every matrix $L$ having the first two columns in common with $L_{1}$.

We show that given $V_{k}$ determined by the pencil $\left[\left(a_{1} t\right)^{n},\left(a_{2} t\right)^{n}\right]$, and $(q t)^{k}=\left(t t_{1}\right)\left(t t_{2}\right) \cdots\left(t t_{k}\right)$, any $k$-ic factor of $\left(a_{1} t\right)^{n}$, we can find a matrix $L$ such that the point $T\left(t_{1}, t_{2}, \cdots, t_{k}\right)$ is on $\Gamma_{s}$ for every set $M_{(\mathrm{s})}^{n-k+1}$, but is on no curve residual to $V_{k}$.

If $k=2, \Gamma_{s}$ and $V_{k}$ are identical, so that the theorem is obvious. If $k=3$, $L$ is of the form $\left(a_{1} a_{2} a_{3} a_{4}\right) . \Gamma_{s}$ is given by the simultaneous vanishing either of $\left(a_{1} a_{2} a_{3}\right),\left(a_{1} a_{2} a_{4}\right)$, or of the dual $c$-matrices, $\left[c_{4} c_{5} \cdots c_{n+1}\right],\left[c_{3} c_{5} \cdots c_{n+1}\right]$. From the latter representation its order is found to be $(n-2)^{2} . V_{k}$ is represented by $\left(a_{1} a_{2}\right) \equiv 0$, or $\left[c_{3} c_{4} \cdots c_{n+1}\right] \equiv 0 ; V_{\Delta}$ by $\left(a_{1} a_{2} a_{3} a_{4}\right) \equiv 0$, or $\left[c_{5} c_{6} \cdots c_{n+1}\right] \equiv 0$. Their orders are respectively $C_{n-1, n-3}, C_{n-2, n-4}$. Since the order of $\Gamma_{s}$ is precisely the sum of those of $V_{k}, V_{\Delta}$, our theorem is proved for this case if we can show that a matrix can be found for which $T$ is not on $V_{\Delta}$. For $k>3$ we determine the conditions to be satisfied if $T$ is on a curve residual to $V_{k}$ and $V_{\Delta}$ in $\Gamma_{s} . \Gamma_{s}$ is given by the vanishing of $k-1 a$-matrices, or the $k-1$ dual $c$-matrices:

(2a) $\left(a_{1} a_{2} a_{3} \cdots a_{k}\right),\left(a_{1} a_{2} a_{4} \cdots a_{k+1}\right), \cdots,\left(a_{1} a_{2} a_{k+1} \cdots a_{2 k-2}\right) \equiv 0$,

(2b) $\left[c_{k+1} \cdots c_{n+1}\right],\left[c_{k+2} \cdots c_{n+1} c_{3}\right], \cdots,\left[c_{2 k-1} \cdots c_{n+1} c_{3} \cdots c_{k}\right] \equiv 0$.

It is made up of $V_{k}$, given by $\left[c_{3} c_{4} \cdots c_{n+1}\right] \equiv 0$, and of irreducible curves each of which is on at least one of the manifolds:

$$
\left[c_{k+2} \cdots c_{n+1}\right],\left[c_{k+3} \cdots c_{n+1} c_{3}\right], \cdots,\left[c_{2 k-1} \cdots c_{n+1} c_{3} \cdots c_{k-1}\right\rfloor \equiv 0 .
$$


For consider an irreducible curve $V^{\prime}$ which is part of $\Gamma_{s}$, and for which $\left[c_{k+2} \cdots c_{n+1}\right] \not \equiv 0$. By Theorem $1,\left[c_{k+1} \cdots c_{n+1} c_{3}\right] \equiv 0$ for $V^{\prime}$. If none of (3) is satisfied, repeated applications of Theorem 1 prove that for the points of $V^{\prime}$, $\left[c_{k+1} \cdots c_{n+1} c_{3} \cdots c_{k}\right] \equiv 0$; thus $V^{\prime}$ coincides with all or part of $V_{k}$. Since no part of $V_{k}$ can figure multiply in $\Gamma_{s}$ [cf. Theorem 3], a point $T$ on a curve residual to $V_{k}$ must therefore make vanish one of the matrices of (3), say $\left[c_{k+2} \cdots c_{n+1}\right]$. Let us further suppose that $T$ is not on $V_{\Delta}$, so that $\left[c_{2 k-1} \cdots c_{n+1}\right] \not \equiv 0$. Then the polar with respect to $(q t)^{k}$ of a linear combination of $\left(c_{k+2} t\right)^{n}, \cdots,\left(c_{2 k-2} t\right)^{n}$ is either identically zero or is the polar of a linear combination of $\left(c_{2 k-1} t\right)^{n}, \cdots,\left(c_{n+1} t\right)^{n}$. This means that in $S_{n}$ the $S_{n-k+2}$ $\left[\left(t t_{1}\right)^{n}, \cdots,\left(t t_{k}\right)^{n},\left(c_{2 k-1} t\right)^{n}, \cdots,\left(c_{n+1} t\right)^{n}\right]$ meets the $S_{k-4}\left[\left(c_{k+2} t\right)^{n}, \cdots,\left(c_{2 k-2} t\right)^{n}\right]$ in a point.

We now build a matrix $L$ of the desired type. The hyperplane $\left(a_{1} t\right)^{n}$ intersects the norm-curve $N^{k}$ in $t_{1}, \cdots, t_{k}$, and is intersected by $\left(a_{2} t\right)^{n}$ in an $S_{n-2}$. In this $S_{n-2}$ we choose $n-2 k+3$ points $\left(c_{2 k-1} t\right)^{n}, \cdots,\left(c_{n+1} t\right)^{n}$, independent of each other and of $\left(t t_{1}\right)^{n}, \cdots,\left(t t_{k}\right)^{n}$. This insures that $T$ is not on $V_{\Delta}$, and if $k=3$, we may choose for $\left(c_{3} t\right)^{n},\left(c_{4} t\right)^{n}$ any two further independent points in the $S_{n-2}$. If $k>3$, we proceed as follows: The points $\left(t t_{1}\right)^{n}, \cdots,\left(t t_{k}\right)^{n}$, $\left(c_{2 k-1} t\right)^{n}, \cdots,\left(c_{n+1} t\right)^{n}$, determine an $S_{n-k+2}$. In the $S_{n-2}$ of $\left(a_{1} t\right)^{n}$ we take an $S_{2 k-5}$ meeting the $S_{n-k+2}$ in an $S_{k-2}$. In the $S_{2 k-5}$ but outside the $S_{k-2}$ we choose $2 k-4$ independent points $\left(c_{3} t\right)^{n}, \cdots,\left(c_{2 k-2} t\right)^{n}$, such that the $S_{k-4}$ determined by any $k-3$ will not intersect the $S_{k-2}$. Then $T$ cannot be on any curve residual to $V_{k}$ in a $\Gamma_{s}$. We take for $\left(c_{1} t\right)^{n},\left(c_{2} t\right)^{n}$ any two further independent $n$-ics, and from these $c$ 's the set of $a$ 's (including $a_{1}, a_{2}$ ) may be determined.

THEOREM 5. If a point is of multiplicity $m$ for all $\Gamma_{s}$ 's determined from every matrix $L$, it is of multiplicity $m$ for $V_{k}$.

TheOREM 6. If an $n$-ic of the pencil contains $k$ pairs of double roots $t_{1}=t_{2}=s_{1}$, $t_{3}=t_{4}=s_{2}, \cdots, t_{2 k-1}=t_{2 k}=s_{k}$, then the point $\left(s_{1}, s_{2}, \cdots, s_{k}\right)$ is a double point for the surface $M^{n-k+1}$.

Taking the particular $n$-ic for $\left(a_{1} t\right)^{n}$ and recalling the equation (1) for $M^{n-k+1}$, we see that the $k$ lines of type $t_{1}=s_{1}, t_{2}=s_{2}, \cdots, t_{k-1}=s_{k-1}$ meet the surface here in two coincident points. If this is not a double point of the surface, the $k$ lines must lie in a common tangent hyperplane. A point of such a line is $\left(t s_{1}\right)\left(t s_{2}\right) \cdots\left(t s_{k-1}\right)\left(t t^{\prime}\right)$, where $t^{\prime}$ may vary. The $k$-ic giving the intersections of the common tangent hyperplane with the normcurve must be apolar to this $k$-ic and so may be written

$$
(\eta t)^{k} \equiv \sum_{i=1}^{k-1} \lambda_{i}\left(t s_{i}\right)^{k}+\lambda_{k}\left(t t^{\prime}\right)^{k} .
$$


But if $(\eta t)^{k}$ may also be written

$$
(\eta t)^{k} \equiv \sum_{i=1}^{k-1} \mu_{i}\left(t s_{i}\right)^{k}+\mu_{k}\left(t t^{\prime \prime}\right)^{k},
$$

we must have $\lambda_{k}=\mu_{k}=0$, since there is no identity connecting $k$ th powers of $k+1$ linear forms. Thus the hyperplane is a linear combination of the hyperplanes $\left(t s_{1}\right)^{k},\left(t s_{2}\right)^{k}, \cdots,\left(t s_{k-1}\right)^{k}$. Similarly it is a linear combination of any $k-1$ of the hyperplanes $\left(t s_{1}\right)^{k}, \cdots,\left(t s_{k}\right)^{k}$. Thus

$$
\sum_{i=1}^{k-1} \lambda_{i}\left(t s_{i}\right)^{k} \equiv \sum_{j=2}^{k} \mu_{j}\left(t s_{j}\right)^{k},
$$

and $\lambda_{1}=\mu_{k}=0$. Likewise all the other $\lambda$ 's are zero. Hence no such hyperplane exists, and $\left(s_{1}, s_{2}, \cdots, s_{k}\right)$ is a double point of the surface.

TheOREM 7. An $S_{k-1}$ of the normcurve $N^{k}$ meets the $M^{n-k+1}$ in an $\bar{M}^{n-k+1}$ of similar type determined by a linear system $\Sigma_{k-2}$ of $(n-1)$-ics related to the normcurve $N^{k-1}$ in $S_{k-1}$.

Let the hyperplane $S_{k-1}$ have the parameter $t_{1}$ on $N^{k}$. It meets $M^{n-k+1}$ in a locus of points with fixed coordinate $t_{1}$. The $(k-1)$-ads giving the other coordinates are obtained after factoring $\left(t t_{1}\right)$ from a system $\left(\lambda_{1}, \cdots, \lambda_{k}\right)$, where $\sum_{i=1}^{k} \lambda_{i}\left(a_{i} t_{1}\right)^{n}=0$. Due to this relation we have a linear system $\Sigma_{k-2}$ determined by $k-1(n-1)$-ics. Using also the theorem that the $S_{k-1}$ 's of a normcurve $N^{k}$ cut out on a fixed hyperplane of $N^{k}$ the $S_{k-2}$ 's of an $N^{k-1}$ in the hyperplane, we may set up for $\bar{M}^{n-k+1}$ an equation similar to (1). By continuing this process, we arrive at the general theorem:

THEOREM 8. The $S_{k-r}(r=1,2, \cdots, k-2)$ which is the intersection of $r$ hyperplanes $t_{1}, t_{2}, \cdots, t_{r}$ of $N^{k}$ meets the $M^{n-k+1}$ in an $\bar{M}^{n-k+1}$ of similar type determined by a system $\Sigma_{k-r-1}$ of $(n-r)$-ics related to the normcurve $N^{k-r}$ in the $S_{k-r}$.

THEOREM 9. If the pencil contains an $n$-ic $\left(t s_{1}\right)\left(t s_{1}\right)\left(t s_{2}\right)\left(t s_{2}\right) \cdots\left(t s_{j}\right)\left(t s_{j}\right)$ $\left(t t_{1}\right)\left(t t_{2}\right) \cdots\left(t t_{n-2 j}\right)$, then at any point $P_{r l}\left(s_{1}, s_{2}, \cdots, s_{r}, s_{r+1}, s_{r+1}, \cdots, s_{r+l}\right.$, $\left.s_{r+l}, t_{1}, t_{2}, \cdots, t_{k-r-2 l}\right)$, the space $S_{r}$ determined by the intersection of hyperplanes $s_{r+1}, s_{r+1}, \cdots, s_{r+l}, s_{r+l}, t_{1}, t_{2}, \cdots, t_{k-r-2 l}$ is tangent to $M^{n-k+1}$ (the intersection of $s_{i}$ with $s_{i}$ is the $S_{k-2}$ of $N^{k}$ at $s_{i}$ ).

For by Theorems 7 and 8 this $S_{r}$ meets $M^{n-k+1}$ in an $\bar{M}^{n-k+1}$ with a double point at $P_{r l}$. Hence any line in $S_{r}$ through $P_{r l}$ is tangent to $M^{n-k+1}$ at that point.

THEOREM 10. The point $P_{r l}$ of Theorem 9 is a point of multiplicity $2^{r-1}$ at least for all $\Gamma_{s}$ 's determined from every matrix $L$. 
If $r=k$, the point is a double point for all $M^{n-k+1}$ 's [cf. Theorem 6], and is therefore of multiplicity $2^{k-1}$ at least for the curve determined by any $k-1$ of the $M^{n-k+1}$ 's. If $r<k, P_{r l}$ must be a simple point for the general $M^{n-k+1}$. However, the tangent hyperplanes to the $M^{n-k+1}$ 's at this point have a common $S_{r}$. Hence we may substitute for the original $M^{n-k+1}$ 's of a set $M_{(\mathrm{z})}^{n-k+1}$ a system of $k-1$ independent $M^{n-k+1}$ 's in which $r-1$ have double points at $P_{r l}$. Their intersection has therefore at $P_{r l}$ a point of multiplicity $2^{r-1}$ at least ([4], p. 191).

THEOREM 11. The point $P_{r l}$ above described is a point of multiplicity exactly $2^{r-1}$ for $V_{k}$.

That the multiplicity is at least $2^{r-1}$ is evident from Theorems 5 and 10 . That $V_{k}$ has no higher multiplicity at $P_{r l}$ is shown by the fact that any hyperplane $s_{i}(i \leqq r)$ meets it here in only $2^{r-1}$ points.

We finally want to prove

THEOREM 12. The restriction $k \leqq(n+3) / 2$ imposed on the curve $V_{k}$ in this section is valid for the generic curves $\left[W_{p}, S_{p}\right]$ of $W_{p}$.

The curves meant are those cut out on $W_{p}$ by $(p+1)$-secant $S_{p}$ 's of $N^{2 p-1}$ as defined in [1], \$5. They are related to a pencil of $(2 p-j)$-ics containing one member with $p-j-1$ double roots $(-2 \leqq j \leqq p-1)$. For $j \geqq 0$, they are involution curves determined by $(p-j)$-ics of the pencil (cf. [1], $\$ \$ 4,6,(4)$, (7)); for $j=-2,-1$, they are projections of such curves (cf. [1], $\$ 6,(9)$ ). Since $p-j \leqq(2 p-j+3) / 2$ for $j \geqq-3$, the restriction is satisfied. Furthermore, for $j \leqq 3$, the restriction is also satisfied for the involution curve determined from the $p$-ics of the pencil, which is a Cremona transform of the generic curve (cf. [1], §6, (11)).

5. Proof of the identity (3) in $\$ 3$. The identity is obviously true for $j=0$. In the following proof we assume $n \geqq 2 j, j \geqq 1, n \geqq k \geqq 1$. Wherever they occur, we take $C_{0,0}=1$ and $C_{a, b}=0$, if $a<b$. These conditions insure that the terms of the right-hand member involve true binomial coefficients and are restrictions obviously imposed by the geometric aspects of our problem. We have

$$
\begin{aligned}
\sum_{r=0} & \sum_{l=0}^{j-r} C_{j, r} C_{j-r, l} C_{n-2 j, k-r-2 l}(k r-r-2 l) 2^{r-1} \\
\quad= & j \sum_{r=0}^{j-1} 2^{r} \sum_{l=0}^{j-r-1} \frac{(j-1) !}{r ! l !(j-r-l-1) !}\left[C_{n-2 j, k-r-2 l-1}(k-1)-C_{n-2 j, k-r-2 l-2}\right] .
\end{aligned}
$$

If in

$$
\sum_{r=0}^{j-1} 2 r \sum_{l=0}^{j-r-1} \frac{(j-1) !}{r ! l !(j-r-l-1) !} C_{n-2 j, k-r-2 l-1}
$$


we put $j-1=j^{\prime}, n-2=n^{\prime}, k-1=k^{\prime}$, we get

$$
\sum_{r=0}^{j^{\prime}} 2^{r} \sum_{l=0}^{j^{\prime}-r} \frac{j^{\prime} !}{r ! l !\left(j^{\prime}-r-l\right) !} C_{n^{\prime}-2 j^{\prime}, k^{\prime}-r-2 l} .
$$

Expanding and rearranging (2) in the form $\sum_{r=0}^{2 j^{\prime}} c_{r} C_{n^{\prime}-2 j^{\prime}, k^{\prime}-r}$, we have

$$
c_{r}=\sum_{i} 2^{r-2 i} C_{j^{\prime}, r-i} C_{r-i, i}, \text { for } r \leqq j^{\prime},
$$

where $i=0,1,2, \cdots,(r-1) / 2$ or $r / 2$, according as $r$ is odd or even;

$$
c_{r}=\sum_{i} 2^{2 j^{\prime}-r-2 i} C_{j^{\prime}, 2 j^{\prime}-r-i} C_{2 j^{\prime}-r-i, i}, \text { for } r>j^{\prime},
$$

where $i=0,1,2, \cdots,\left(2 j^{\prime}-r-1\right) / 2$ or $\left(2 j^{\prime}-r\right) / 2$, according as $r$ is odd or even. Since $2 j^{\prime}-r<j^{\prime}$ if $r>j^{\prime}$, both forms are of similar type. Using the identity $C_{j^{\prime}, r-i} C_{r-i, i}=C_{j^{\prime}, i} C_{j^{\prime}-i, r-2 i}$ (cf. [5], 20 , p. 178), we transform (3a) into (3a) $)^{\prime} \quad c_{r}=\sum_{i} 2^{r-2 i} C_{j^{\prime}, i} C_{j^{\prime}-i, r-2 i}, \quad i=0,1,2, \cdots,(r-1) / 2$, or $r / 2$.

We now prove that $(3 \mathrm{a})^{\prime}$ gives us the binomial coefficient $C_{2 j^{\prime}, r}$. Let $2 j^{\prime}$ objects be divided into $j^{\prime}$ pairs. To a set of $r$ taken, let $i$ pairs $(i \leqq r / 2)$ contribute both their members, while $r-2 i$ pairs contribute one member each. The $i$ pairs may be chosen in $C_{j^{\prime}, i}$ ways, the $r-2 i$ pairs in $C_{j^{\prime}-i, r-2 i}$ ways; the particular member from each of the latter in 2 ways. The total number of ways as $i$ varies from 0 to $(r-1) / 2$ or $r / 2$ is the $c_{r}$ given by ${ }_{-}^{r}(3 a)^{\prime}$. Similarly the $c_{r}$ of (3b) can be proved equal to $C_{2 j^{\prime}, 2 j^{\prime}-r}=C_{2 j^{\prime}, r}$. Thus (2)_becomes (cf. $[5], 6^{0}$, p. 178)

$$
\sum_{r=0}^{2 j^{\prime}} C_{2 j^{\prime}, r} C_{n^{\prime}-2 j^{\prime}, k^{\prime}-r}=C_{n^{\prime}, k^{\prime}}=C_{n-2, k-1}
$$

By a similar process we prove

$$
\sum_{r=0}^{j-1} 2^{r} \sum_{l=0}^{j-r-1} \frac{(j-1) !}{r ! l !(j-r-l-1) !} C_{n-2 j, k-r-2 l-2}=C_{n-2, k-2} .
$$

Hence

$$
\begin{aligned}
\sum_{r=0}^{j} \sum_{l=0}^{j-r} C_{j, r} C_{j-r, l} C_{n-2 j, k-r-2 l}(k r-r-2 l) 2^{r-1} & =j\left[C_{n-2, k-1}(k-1)-C_{n-2, k-2}\right] \\
& =j(n-k-1) C_{n-2, k-2},
\end{aligned}
$$

which was to be proved.

6. Special involution curves for $k=2$. We want to know what situations arise when some or all of the $j$ double roots in the special $n$-ic $[\S 2,3,4]$ 
coincide, as may occur for the involution curves of $W_{p}$. Definite results can easily be obtained for $k=2$. In the special $n$-ic let $2 k_{1}$, of the roots coincide in $t_{1}, 2 k_{2}$ in $t_{2}, \cdots, 2 k_{m}$ in $t_{m}$, while $n-2 \sum_{i=1}^{m} k_{i}$ of the roots remain simple. If $x_{0}$ corresponds to the special $n$-ic, then as $x$, very near $x_{0}$, turns about $x_{0}$, the roots of the $n$-ic corresponding to $x$ are permuted according to the permutation

(1) $S=\left(t_{11} t_{12} \cdots t_{1 k_{1}}\right)\left(t_{21} t_{22} \cdots t_{2 k_{2}}\right) \cdots\left(t_{m_{1}} t_{m_{2}} \cdots t_{m k_{m}}\right)\left(\tau_{1}\right)\left(\tau_{2}\right) \cdots\left(\tau_{n-2 \Sigma k_{i}}\right)$.

A corresponding permutation $\Sigma$ takes place among the $C_{n, 2}$ pairs of $t$ 's and $\tau$ 's chosen from the roots of the $n$-ic. As $x$ approaches $x_{0}$, a letter $t_{i}$ chosen from the $i$ th cycle approaches the point $P_{i}$ of $N^{2}$, and $\tau_{h}$ approaches the point $Q_{h}$ of $N^{2}$. Likewise a pair $\left(t_{i} t_{i}^{\prime}\right)$ chosen from the same cycle approaches the point $\pi_{i}$ of $V_{2}$, a pair $\left(t_{i} t_{l}\right)$ from different cycles approaches the point $\pi_{i l}$ of $V_{2}$, a pair $\left(t_{i} \tau_{h}\right)$ approaches the point $\rho_{i h}$ of $V_{2}$, and a pair $\left(\tau_{h} \tau_{s}\right)$ approaches the point $\sigma_{h s}$ of $V_{2}$.

We first seek what contribution $P_{i}$ makes to $\eta_{\boldsymbol{s}}$ due to permutations among the $k$-ads $\left(t_{i} t_{l}\right)$. Let $d_{i l}$ be the lowest common multiple of $2 k_{i}, 2 k_{l}$. The permutation $S^{2 k_{i}}$ carries $t_{i}$ around $P_{i}$. The permutation induced by this among the $t$ 's of the $l$ th cycle is of period $d_{i l} / 2 k_{i}$, and so consists of $4 k_{i} k_{l} / d_{i l}$ cycles of $d_{i l} / 2 k_{i}$ letters each. Hence the pairs $\left(t_{i} t_{l}\right)$ on $t_{i}$ are permuted likewise in $4 k_{i} k_{l} / d_{i l}$ cycles of $d_{i l} / 2 k_{i}$ pairs each. The same argument is followed when we consider a point $Q_{h}$ and pairs containing $\tau$ 's. Hence

$$
\begin{gathered}
\eta_{s}=\sum_{i, l}\left[4 k_{i} k_{l} / d_{i l}\right]\left[d_{i l}\left(1 / 2 k_{i}+1 / 2 k_{l}\right)-2\right] \\
+\left[n-2 \sum_{i} k_{i}\right]\left[\sum_{i}\left(2 k_{i}-1\right)\right],
\end{gathered}
$$

where $i, l$ take all integral values from 1 to $m$, and where $i<l$ in each term of the first summation. We next determine $\eta_{s}^{\prime}$. No points $\pi_{i l}, \rho_{i h}, \sigma_{h s}$ of $V_{2}$ can be branch points, since the corresponding permutations are powers of $S$ which leave fixed the $t$ 's and $\tau$ 's involved in each case. If $\pi_{i}$ is a branch point, the point $\left(t_{i} t_{i}^{\prime}\right)$ moving around it must reach its original position by an interchange of $t_{i}, t_{i}^{\prime}$. The corresponding permutation among the letters of the $i$ th cycle must therefore be of period two and consist of $k_{i}$ cycles. As $\left(t_{i} t_{i}^{\prime}\right)$ moves around $\pi_{i}$, it passes successively through the points represented by these cycles. Hence each $\pi_{i}$ counts once as a branch point and

$$
\eta_{s}^{\prime}=m \text {. }
$$

Finally there are in the pencil of $n$-ics $2(n-1)-\sum_{i=1}^{m}\left(2 k_{i}-1\right)$ members with a double root each. Their contribution is 


$$
\eta_{0}-\eta_{0}^{\prime}=\left[2(n-1)-\sum_{i=1}^{m}\left(2 k_{i}-1\right)\right][n-3] .
$$

From (2), (3), and (4), we may easily determine $p_{2}$ by Zeuthen's formula.

In concrete cases it is of greater advantage to know at once the effect on $V_{2}$ 's genus of the points $\pi_{i}, \pi_{i l}, \rho_{i h}, \sigma_{h s}$. Since a $\sigma_{h s}$ is an ordinary simple point of $V_{2}$, and a $\rho_{i h}$ is a simple point with a $2 k_{i}$-point contact tangent $\tau_{h}$, our attention is concentrated on points $\pi_{i}$ and $\pi_{i l}$. The effect of either type is independent of the roots of the $n$-ic not appearing in the pair determining the point. Thus $\pi_{i}$ is best studied by considering a pencil of $2 k_{i}$-ics which contains one member with a $2 k_{i}$-fold root. By (2), (3), and (4), $\eta_{s}=0, \eta_{s}^{\prime}=1, \eta_{0}-\eta_{0}^{\prime}$ $=\left(2 k_{i}-1\right)\left(2 k_{i}-3\right)$. Hence

$$
\eta-\eta^{\prime}=4 k_{i}^{2}-8 k_{i}+2,
$$

and

$$
p_{2}=k_{i}{ }^{2}-3 k_{i}+2 \text {. }
$$

Since $C_{2 k_{i}-2,2}$ is the maximum value of the genus for curves of $C_{2}$ 's order we have proved the theorem:

THEOREM 1. The point $\pi_{i}$ introduced into $V_{2}$, if the pencil contains an $n$-ic with a $2 k_{i}$-fold root, is equivalent to $\left(k_{i}-1\right)^{2}$ ordinary double points in the calculation of the genus.

THEOREM 2. If the pencil of $n$-ics contains a member with one $2 k_{i}$-fold root, and another $2 k_{l}$-fold root, the point $\pi_{i l}$ introduced into $V_{2}$ decreases the genus by the number $M=2 k_{i} k_{l}-k_{i}-k_{l}+f\left(k_{i}, k_{l}\right)$, where $f\left(k_{i}, k_{l}\right)$ is the greatest common divisor of $k_{i}$ and $k_{l}$. Since $M$ may be written $C_{2 k_{l}, 2}+\left[2 k_{l}\left(k_{i}-k_{l}\right)-k_{i}\right.$ $\left.+f\left(k_{i}, k_{l}\right)\right]$, the effect of $\pi_{i l}$ on the genus is that of a $2 k_{l}$-fold point into which is absorbed an extra number of double points equal to the number $N$ in the brackets.

Note the symmetry of $N$ in $k_{l}$ and $k_{i}-k_{l}$. To prove the theorem, we take a pencil of $2\left(k_{i}+k_{l}\right)$-ics containing one member $\left(t s_{i}\right)^{2 k_{i}}\left(t s_{l}\right)^{2 k_{l}}$. Substituting $2 k_{i} k_{l} / f\left(k_{i}, k_{l}\right)$ for $d_{i l}$ in (2), and using also (3), (4), we have

$$
\eta-\eta^{\prime}=4 k_{i}{ }^{2}+8 k_{i} k_{l}+4 k_{l}{ }^{2}-4 k_{i}-4 k_{l}-4 f\left(k_{i}, k_{l}\right)-2 .
$$

Zeuthen's formula gives us

$$
p_{2}=k_{i}{ }^{2}+2 k_{i} k_{l}+k_{l}{ }^{2}-2 k_{i}-2 k_{l}-f\left(k_{i}, k_{l}\right)+1 \text {. }
$$

The maximum genus for a curve of $V_{2}$ 's order is $C_{2 k_{i}+2 k_{l}-2,2}$. Allowing for the reductions $\left(k_{i}-1\right)^{2},\left(k_{l}-1\right)^{2}$ in $p_{2}$ due to $\pi_{i}, \pi_{l}$ respectively, we get $2 k_{i} k_{l}-k_{i}$ $-k_{l}+f\left(k_{i}, k_{l}\right)$ as the reduction due to $\pi_{i l}$.

We now construct several curves in order to give concrete examples of the 
multiple points. Let us first consider the pencil $\lambda_{0}\left(t t_{1}\right)^{8} \lambda_{1}(\alpha t)^{8}$, where $t_{1}=0$, $(\alpha t)^{8}=\alpha_{0} t^{8}+\alpha_{1} t^{7}+\alpha_{2} t^{6}+\alpha_{3} t^{5}+\alpha_{4} t^{4}+\alpha_{5} t^{3}+\alpha_{6} t^{2}+\alpha_{7} t+\alpha_{8}$. The equation of $V_{2}^{7}$ is

$$
\left|\begin{array}{cc}
t^{8} & (\alpha t)^{8} \\
t^{\prime 8} & \left(\alpha t^{\prime}\right)^{8}
\end{array}\right| \cdot \frac{1}{t-t^{\prime}}=0 .
$$

If we put $x_{0}=t t^{\prime}, x_{1}=t+t^{\prime}, x_{2}=1$, the point $\left(t_{1} t_{1}\right)$ on $V_{2}^{7}$ has coordinates $(0,0,1)$; the line $t_{1}$ has the equation $x_{0}=0$; and the equation of $V_{2}{ }^{7}$, arranged in powers of $x_{2}$, becomes

$$
\begin{aligned}
& x_{0}^{3} x_{2}^{3}\left(\alpha_{7} x_{0}+4 \alpha_{8} x_{1}\right)-x_{0}{ }^{2} x_{2}{ }^{2}\left(\alpha_{5} x_{0}{ }^{3}+3 \alpha_{6} x_{0}{ }^{2} x_{1}+6 \alpha_{7} x_{0} x_{1}{ }^{2}+10 \alpha_{8} x_{1}{ }^{3}\right) \\
& +x_{0} x_{2}\left(\alpha_{3} x_{0}{ }^{5}+2 \alpha_{4} x_{0}{ }^{4} x_{1}+3 \alpha_{5} x_{0}{ }^{3} x_{1}{ }^{2}+4 \alpha_{6} x_{0}{ }^{2} x_{1}{ }^{3}+5 \alpha_{7} x_{0} x_{1}{ }^{4}+6 \alpha_{8} x_{1}{ }^{5}\right) \\
& -\alpha_{1} x_{0}{ }^{7}-\alpha_{2} x_{0}{ }^{6} x_{1}-\alpha_{3} x_{0}{ }^{5} x_{1}{ }^{2}-\alpha_{4} x_{0}{ }^{4} x_{1}{ }^{3}-\alpha_{5} x_{0}{ }^{3} x_{1}{ }^{4}-\alpha_{6} x_{0}{ }^{2} x_{1}{ }^{5} \\
& -\alpha_{7} x_{0} x_{1}{ }^{6}-\alpha_{8} x_{1}{ }^{7}=0 .
\end{aligned}
$$

The point $\left(t_{1} t_{1}\right)$ is shown to be a fourfold point on $V_{2}^{7}$. The line $t_{1}$, counting three times as a tangent line, meets $V_{2}^{7}$ in seven points here. If we put $x_{0}=y$, $x_{1}=x, x_{2}=1$, and use Newton's method of approximation, we find that in the neighborhood of the origin $\left(t_{1} t_{1}\right)$, the curve has three branches of type $y=A x^{2}$ and one of type $y=B x$. On a slight dislocation of the configuration every pair of the three former branches intersect in two points, while the fourth intersects the others in one point each. This accounts for the nine double points absorbed at $\left(t_{1} t_{1}\right)$.

We next consider the pencil $\lambda_{0}\left(t t_{1}\right)^{8}\left(t t_{9}\right)^{2}+\lambda_{1}(\beta t)^{10}$, where $t_{1}=0, t_{9}=\infty$. If we again transform to homogeneous coordinates, the point $\left(t_{1} t_{9}\right)$ has coordinates $(0,1,0)$; the equations of $t_{1}, t_{9}$ are $x_{0}=0, x_{2}=0$ respectively; and the equation of $V_{2}{ }^{9}$, arranged in powers of $x_{1}$, becomes

$$
\begin{aligned}
& \beta_{10} x_{1}{ }^{7} x_{2}{ }^{2}+\beta_{9} x_{0} x_{1}{ }^{6} x_{2}{ }^{2}+x_{1}{ }^{5} x_{2}{ }^{2}\left(\beta_{8} x_{0}{ }^{2}-6 \beta_{10} x_{0} x_{2}\right)+x_{1}{ }^{4} x_{2}{ }^{2}\left(\beta_{7} x_{0}{ }^{3}-5 \beta_{9} x_{0}{ }^{2} x_{2}\right) \\
& +x_{1}{ }^{3} x_{2}{ }^{2}\left(\beta_{6} x_{0}{ }^{4}-4 \beta_{8} x_{0}{ }^{3} x_{2}+10 \beta_{10} x_{0}{ }^{2} x_{2}{ }^{2}\right)+x_{1}{ }^{2} x_{2}{ }^{2}\left(\beta_{5} x_{0}{ }^{5}-3 \beta_{7} x_{0}{ }^{4} x_{2}+6 \beta_{9} x_{0}{ }^{3} x_{2}{ }^{2}\right) \\
& -x_{1}\left(\beta_{0} x_{0}{ }^{8}-\beta_{4} x_{0}{ }^{6} x_{2}{ }^{2}+2 \beta_{6} x_{0}{ }^{5} x_{2}{ }^{3}-3 \beta_{8} x_{0}{ }^{4} x_{2}{ }^{4}+4 \beta_{10} x_{0}{ }^{3} x_{2}{ }^{5}\right) \\
& -\beta_{1} x_{0}{ }^{8} x_{2}+\beta_{3} x_{0}{ }^{7} x_{2}{ }^{2}-\beta_{5} x_{0}{ }^{6} x_{2}{ }^{3}+\beta_{7} x_{0}{ }^{5} x_{2}{ }^{4}-\beta_{9} x_{0}{ }^{4} x_{2}{ }^{5}=0 .
\end{aligned}
$$

The point $\left(t_{1} t_{9}\right)$ is a double point; the two tangents coincide in $t_{9}$, which meets $V_{2}{ }^{9}$ here in eight points. Putting $x_{0}=x, x_{1}=1, x_{2}=y$, we find by Newton's approximation that the two branches at the origin $\left(t_{1} t_{9}\right)$ are of types $y=A x^{4}$, $y=-A x^{4}$, respectively. Each branch touches the tangent at a point of undulation. If the coefficients of $V_{2}{ }^{9}$ 's equation were very slightly changed, the two branches would intersect in the four double points which are absorbed at $\left(t_{1} t_{9}\right)$.

We finally take the pencil $\lambda_{0}\left(t t_{1}\right)^{6}\left(t t_{7}\right)^{4}+\lambda_{1}(\gamma t)^{10}$, where $t_{1}=0, t_{7}=\infty$. The 
point $\left(t_{1} t_{7}\right)$ has coordinates $(0,1,0)$; the equations for $t_{1}, t_{7}$ are $x_{0}=0, x_{2}=0$ respectively, and $V_{2}{ }^{9}$ 's equation, arranged in powers of $x_{1}$, reads

$$
\begin{aligned}
& \gamma_{10} x_{1}{ }^{5} x_{2}{ }^{4}+\gamma_{9} x_{0} x_{1}^{4} x_{2}{ }^{4}-x_{0} x_{1}{ }^{3}\left(\gamma_{0} x_{0}{ }^{5}-\gamma_{8} x_{0} x_{2}{ }^{4}+4 \gamma_{10} x_{2}{ }^{5}\right) \\
& -x_{0} x_{1}^{2} x_{2}\left(\gamma_{1} x_{0}{ }^{4}-\gamma_{7} x_{0} x_{2}{ }^{3}+3 \gamma_{9} x_{2}^{4}\right)+x_{0}{ }^{2} x_{1} x_{2}\left(2 \gamma_{0} x_{0}{ }^{5}-\gamma_{2} x_{0} x_{2}\right. \\
& \left.+\gamma_{6} x_{0}{ }^{2} x_{2}{ }^{3}-2 \gamma_{8} x_{0} x_{2}{ }^{4}+3 \gamma_{10} x_{2}{ }^{5}\right)+\gamma_{1} x_{0}{ }^{7} x_{2}{ }^{2}-\gamma_{3} x_{0}{ }^{6} x_{2}{ }^{3}+\gamma_{5} x_{0}{ }^{5} x_{2}{ }^{4} \\
& -\gamma_{7} x_{0} x_{2}{ }^{5}+\gamma_{9} x_{0}{ }^{3} x_{2}{ }^{6}=0 .
\end{aligned}
$$

The point $\left(t_{1} t_{7}\right)$ is a fourfold point on $V_{2}{ }^{9}$, at which the four tangents coincide in $t_{7}$, meeting $V_{2}{ }^{9}$ in six points here. If we put $x_{0}=x, x_{1}=1, x_{2}=y$, we find by Newton's method that the curve has at the origin $\left(t_{1} t_{7}\right)$ two branches of types $y^{2}=A x^{3}, y^{2}=-A x^{3}$ respectively. If we allowed these two cusps to change into interlacing loops, we should bring into evidence the eight double points absorbed at $\left(t_{1} t_{7}\right)$.

For higher values of $k$ the same general method may be employed to determine $\eta_{s}, \eta_{s}^{\prime}$, but the application will naturally involve difficulties due to the greater complexities of these cases.

\section{BIBLIOGRAPHY}

1. A. B. Coble and J. H. Chanler, The geometry of the Weddle manifold $W_{p}$, American Journal of Mathematics, vol. 57 (1935), pp. 183-218.

2. A. Brill. Über Elimination aus einem gewissen System von Gleichungen, Mathematische Annalen, vol. 5 (1872), pp. 378-396.

3. M. Pieri, Sull'ordine della varietd̀ generata da più sistemi lineari omografici, Rendiconti del Circolo Matematico di Palermo, vol. 11 (1897), pp. 58-63.

4. E. Bertini, Einführung in die projective Geometrie Mehrdimensionaler Räume, 1924.

5. A. B. Coble, Restricted systems of equations, American Journal of Mathematics, vol. 36 (1914), pp. $167-186$.

UNIVERSITY OF ILLINOIS, URBANA, ILL. 